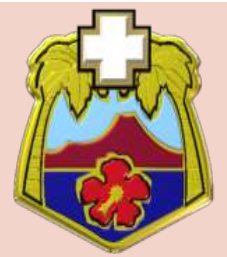

\title{
A Comprehensive Perioperative Regional Anesthesia and Acute Pain Pathway is Associated with Enhanced Recovery after Outpatient Anterior Cruciate Ligament Reconstruction \\ Michael Patzkowski, MD¹, Krista Highland, PhD²,3, Jeanne Patzkowski, MD ${ }^{1}$
}

(1) Tripler Army Medical Center, Honolulu, HI, USA; (2) Defense and Veterans Center for Integrative Pain Management,

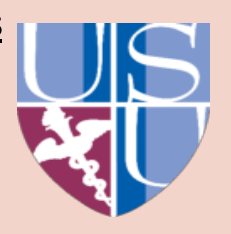

Background and Aims : Outpatient anterior cruciate ligament reconstruction (ALCR) surgery is one of the most common sports orthopedics procedures performed in the world. The present study sought to determine if a comprehensive regional anesthesia and acute pain pathway (RAAPP) was associated with improved patient outcomes prior to discharge.

Methods: Retrospective data of all primary ALCR cases in a facility $(\mathrm{N}=103)$ were divided into Pre- $(\mathrm{n}=55)$ and Post$(n=48)$ RAAPP groups. Bivariate tests assessed group differences in demographic and clinical factors.

Generalized linear models (GLMs) examined effect of group on highest post-operative numeric pain rating (NRS) pain scores, post-anesthesia care unit recovery time, and opioid utilization [morphine-equivalence dose (MED)] from surgery through discharge, while controlling for relevant covariates. Significance level was adjusted for three outcomes at $p<.017$.

Table 1. Analgesia Pathway Group Differences Across Patient Characteristics

\begin{tabular}{lcccc}
\hline & $\begin{array}{c}\text { Pre-RAAPP } \\
(\mathbf{n}=\mathbf{5 5})\end{array}$ & $\begin{array}{c}\text { Post-RAAPP } \\
(\mathbf{n = 4 8})\end{array}$ & SMD & $\begin{array}{c}\text { Significance } \\
\text { level, } \boldsymbol{p}\end{array}$ \\
\hline Age, median [IQR] & $\begin{array}{c}25.00[22.00, \\
31.50]\end{array}$ & $\begin{array}{c}26.00[22.00, \\
32.25]\end{array}$ & 0.003 & 0.75 \\
\hline Male, n (\%) & $42(76.4)$ & $42(87.5)$ & 0.29 & 0.20 \\
\hline $\begin{array}{l}\text { Active-duty Military, n } \\
(\%)\end{array}$ & $39(70.9)$ & $45(93.8)$ & 0.62 & 0.004 \\
\hline $\begin{array}{l}\text { Body Mass Index, median } \\
\text { [IQR] }\end{array}$ & $\begin{array}{c}26.66[24.17, \\
29.20]\end{array}$ & $\begin{array}{c}27.39[24.61, \\
29.72]\end{array}$ & 0.09 & 0.45 \\
\hline $\begin{array}{l}\text { Weight (kg), median } \\
{[\text { IQR] }}\end{array}$ & $\begin{array}{c}85.40[74.65, \\
93.05]\end{array}$ & $\begin{array}{c}83.95[75.62, \\
93.28]\end{array}$ & 0.08 & 0.76 \\
\hline
\end{tabular}

Note: Continuous variables were not normally distributed, and group differences were assessed with Mann-Whitney U tests. Categorical variable differences were assessed with Fisher exact tests. $\mathrm{SMD}=\mathrm{Standardized}$ Mean Difference.

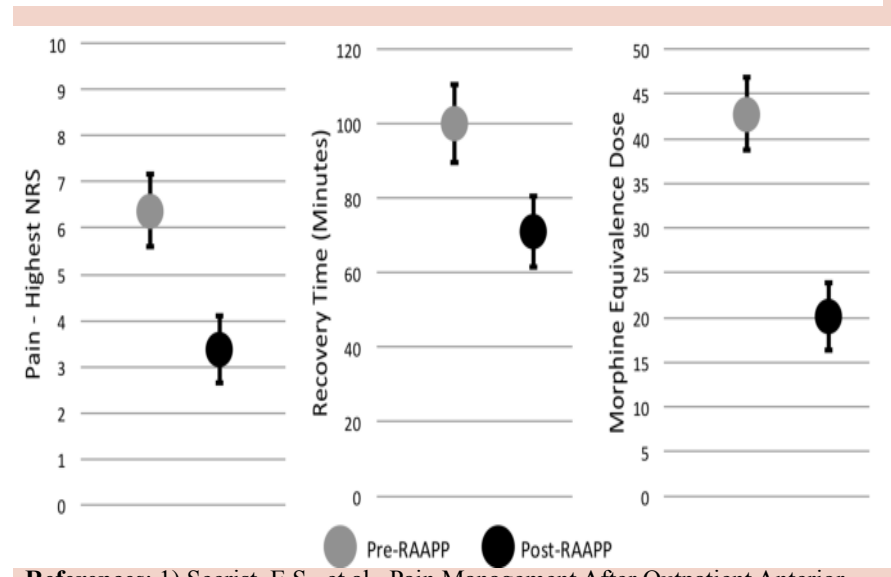

References: 1) Secrist, E.S., et al., Pain Management After Outpatient Anterior Cruciate Ligament Reconstruction: A Systematic Review of RCTs. Am J Sports Med, 2016. 44(9): p. 2435-47. 2) Harbell, M.W., et al.,Combined preoperative femoral and sciatic nerve blockade improves analgesia after anterior cruciate ligament reconstruction: a randomized controlled trial. J Clin Anesth, 2016. Sep; 33: p. 68-74. 3) Anderson, M.J., et al., A Systematic Summary of Systematic Reviews on the Topic of the Anterior Cruciate Ligament. Orthop J Sports Med, 2016. 4(3): p. 2325967116634074.
Results: Post-RAAPP (94\%) had more military service members than Pre-RAAPP $(71 \%, \mathrm{p}<.01)$. Surgical variables were similar except for increased surgical time, preoperative femoral and sciatic nerve blocks, preoperative acetaminophen, gabapentin, celecoxib, and intraoperative dexamethasone in the Post-RAAPP (all $p<$. 05). GLMs controlled for military status and surgical time. Highest post-operative pain NRS scores prior to discharge $[3.38(95 \% \mathrm{Cl} 2.64,4.11)$ vs. $6.38(95 \% \mathrm{Cl} 5.60,7.16)]$, postanesthesia care unit recovery time [ 71 minutes $(95 \% \mathrm{Cl} 61$, $80)$ vs. 100 minutes $(95 \% \mathrm{Cl} 89,110)]$, and total perioperative opioid utilization [20.09 MED $(95 \% \mathrm{Cl} 16.26$, 23.91) vs. $42.74 \mathrm{MED}(95 \% \mathrm{Cl} 38.69,46.80)$ ] were significantly lower in the Post-RAAPP than Pre-RAAPP, respectively (all $p<.001$ ).

Conclusions: Increased utilization of pre-operative regional anesthesia and non-opioid analgesics in ACLR were significantly associated with reduced pain scores, faster early recovery times, and opioid usage prior to discharge.

Table 2. Analgesia Pathway Group Differences Across Perioperative Factors and Analgesia Agents/Procedures

\begin{tabular}{|c|c|c|c|c|}
\hline & $\begin{array}{c}\text { Pre- } \\
\text { RAAPP } \\
(\mathbf{n}=55)\end{array}$ & $\begin{array}{c}\text { Post- } \\
\text { RAAPP } \\
(n=48)\end{array}$ & SMD & $\begin{array}{l}\text { Significance } \\
\quad \text { level, } p\end{array}$ \\
\hline $\begin{array}{l}\text { Operation minutes, median } \\
\text { [IQR] }\end{array}$ & $\begin{array}{c}90.00 \\
{[60.00} \\
129.50]\end{array}$ & $\begin{array}{l}108.50 \\
{[77.50} \\
152.50]\end{array}$ & 0.40 & 0.04 \\
\hline $\begin{array}{l}\text { Graft type, n (\%) } \\
\text { Allograft }\end{array}$ & $\mid 14(25.5)$ & $5(10.4)$ & 0.19 & 0.10 \\
\hline Hamstring Autograft & $\mathrm{t} 21(38.2)$ & $26(54.2)$ & & \\
\hline Patellar Tendon Autograft & $20(36.3)$ & $17(35.4)$ & & \\
\hline $\begin{array}{l}\text { Concomitant surgical procedure, } \\
\mathrm{n}(\%)\end{array}$ & 24 (43.6) & $20(41.7)$ & 0.04 & 1.00 \\
\hline $\begin{array}{l}\text { Femoral nerve block, n (\%) } \\
\text { None }\end{array}$ & $18(32.7)$ & $4(18.2)$ & 1.97 & $<.001$ \\
\hline Administered pre-operatively & $13(23.6)$ & $44(91.7)$ & & \\
\hline Administered post-operatively & $24(43.6)$ & $0(0.0)$ & & \\
\hline $\begin{array}{l}\text { Sciatic nerve block, n (\%) } \\
\text { None }\end{array}$ & 46 (83.6) & $11(22.9)$ & 2.47 & $<0.001$ \\
\hline $\begin{array}{c}\text { Administered pre-operatively } \\
\text { Administered post-operatively }\end{array}$ & $\begin{array}{c}0(0.0) \\
9(16.4)\end{array}$ & $\begin{array}{c}36(75.0) \\
1(2.1)\end{array}$ & & \\
\hline $\begin{array}{l}\text { Adductor canal block, n (\%) } \\
\text { None }\end{array}$ & $\begin{array}{c}55 \\
(100.0)\end{array}$ & $47(97.9)$ & 0.21 & 0.47 \\
\hline $\begin{array}{l}\text { Administered pre-operatively } \\
\text { Administered post-operatively }\end{array}$ & $\begin{array}{l}0(0.0) \\
0(0.0)\end{array}$ & $\begin{array}{l}0(0.0) \\
1(2.1)\end{array}$ & & \\
\hline Pre-operative Medications & & & & \\
\hline Acetaminophen, n (\%) & $0(0.0)$ & $36(75.0)$ & 2.45 & $<0.001$ \\
\hline Gabapentin, n (\%) & $0(0.0)$ & $37(77.1)$ & 2.59 & $<0.001$ \\
\hline Celecoxib, n (\%) & $0(0.0)$ & $36(75.0)$ & 2.45 & $<0.001$ \\
\hline Intra-operative Medications & & & & \\
\hline Dexamethasone, n (\%) & $38(73.1)$ & $46(95.8)$ & 0.66 & 0.002 \\
\hline Ondansetron, (\%) & $51(92.7)$ & $48(100.0)$ & 0.4 & 0.12 \\
\hline Ketamine, $\mathrm{n}(\%)$ & $4(7.3)$ & $10(20.8)$ & 0.39 & 0.08 \\
\hline Ketorolac, n (\%) & $11(20.0)$ & $3(6.2)$ & 0.42 & 0.05 \\
\hline Dexmedetomidine, $\mathrm{n}(\%)$ & $2(3.6)$ & $3(6.2)$ & 0.12 & 0.66 \\
\hline Acetaminophen, n (\%) & $5(9.1)$ & $2(4.2)$ & 0.20 & 0.45 \\
\hline
\end{tabular}

assessed with Mann-Whitney U tests. Categorical variable differences were assessed with Fisher exact tests. SMD=Standardized Mean Difference.

The views expressed in this abstract are those of the author(s) and do not reflect the official policy or position of Tripler Army Medical Center, Uniformed Services University, the Department of the Army, Department of Defense, or the U.S. Government. 